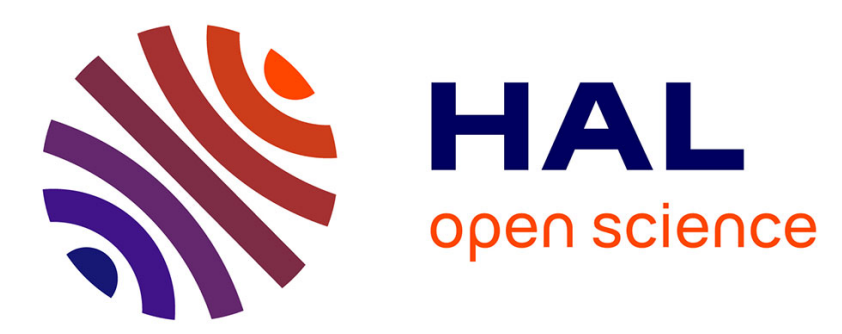

\title{
Mesure du SO2 et des cendres volcaniques avec Iasi
}

Cathy Clerbaux, Maya George, Juliette Hadji-Lazaro, Lieven Clarisse, Daniel Hurtmans, Pierre-François Coheur

\section{To cite this version:}

Cathy Clerbaux, Maya George, Juliette Hadji-Lazaro, Lieven Clarisse, Daniel Hurtmans, et al.. Mesure du SO2 et des cendres volcaniques avec Iasi. La Météorologie, 2011, Août (n74), pp.3541. hal-00665433

\section{HAL Id: hal-00665433 https://hal.science/hal-00665433}

Submitted on 26 Mar 2021

HAL is a multi-disciplinary open access archive for the deposit and dissemination of scientific research documents, whether they are published or not. The documents may come from teaching and research institutions in France or abroad, or from public or private research centers.
L'archive ouverte pluridisciplinaire HAL, est destinée au dépôt et à la diffusion de documents scientifiques de niveau recherche, publiés ou non, émanant des établissements d'enseignement et de recherche français ou étrangers, des laboratoires publics ou privés. 


\title{
Mesure du $\mathrm{SO}_{2}$ et des cendres volcaniques avec IASI
}

\author{
Cathy Clerbaux ${ }^{(1)}$, Maya George ${ }^{(1)}$, Juliette Hadji-Lazaro(1), \\ Lieven Clarisse $^{(2)}$, Daniel Hurtmans ${ }^{(2)}$ et Pierre Coheur ${ }^{(2)}$ \\ (1) LATMOS-IPSL - Université Paris 6 - CNRS/INSU \\ 4, place Jussieu - 75252 Paris Cedex 05 \\ (2) Spectroscopie de l'atmosphère, Service de chimie quantique \\ et photophysique - Université libre de Bruxelles (ULB) - Belgique
}

\section{Résumé}

Les mois de mai et juin 2011 ont été marqués par une succession de trois éruptions volcaniques, mettant en alerte continue les centres responsables de la surveillance des espaces aériens. Le sondeur infrarouge IASI, embarqué sur le satellite MetOp depuis fin 2006, permet de suivre les panaches de $\mathrm{SO}_{2}$ et de cendres associés aux éruptions volcaniques. Cet article illustre les performances de l'instrument pour fournir des informations en temps quasi réel pour l'aviation civile et détaille les méthodes qui ont été mises en ouvre pour calculer les concentrations en gaz et en particules.

\section{Abstract}

Volcanic $\mathrm{SO}_{2}$ and ash measured by IASI

May and June 2011 were marked by three successive volcanic eruptions, putting the dedicated centers responsible for airspace monitoring onto continuous alert. The IASI infrared sounder, flying onboard the MetOp satellite since 2006, can track $\mathrm{SO}_{2}$ and ash plumes associated with volcanic eruptions. This paper describes the performance of the instrument in providing near real-time information for civil aviation. It also details the methods used to derive concentrations of gases and particulates.
(Mount Galunggung). Cet épisode et d'autres similaires ont conduit à mettre au point un système de suivi des éruptions volcaniques afin de permettre une alerte rapide aux avions qui sont amenés à croiser les panaches (voir encadré page suivante). La communauté s'est organisée ; le globe a été divisé en neuf zones dont la surveillance est placée sous la responsabilité d'organismes dédiés, les VAAC (Volcanic Ash Advisory Centers). La figure 1 présente les principales routes aériennes, les chaînes volcaniques actives et les zones de surveillance couvertes par les neuf centres. Le VAAC qui a la charge de la région Europe-Afrique est placé sous la responsabilité de Météo-France à Toulouse.

Figure 1 - Zones géographiques surveillées par les VAAC (pointillés gris), principales routes aériennes (en bleu) et localisation des volcans en activité (en rouge). Source : International Civil Aviation Organisation.

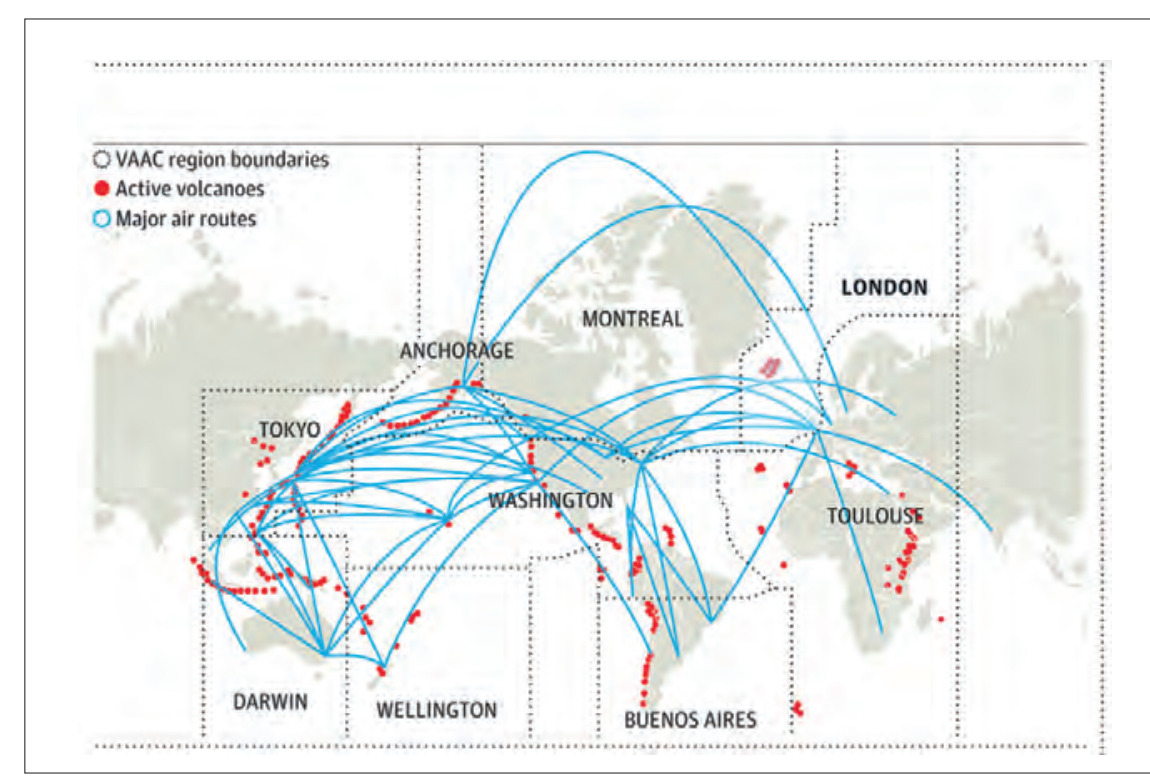


Pendant les heures qui suivent une éruption, les VAAC doivent localiser et prévoir le déplacement des nuages de cendres volcaniques qui représentent un danger potentiel à l'intérieur de leurs zones. Chaque centre s'organise au mieux pour développer les outils et récolter les données nécessaires pour distinguer les nuages de cendres volcaniques des nuages météorologiques (eau, glace) et autres particules, afin de pouvoir caractériser les nuages de cendres (altitude, composition, masse totale de poussière en suspension).

Depuis l'épisode du volcan Eyjafjallajökull en avril-mai 2010, qui a entraîné la plus grande paralysie de l'espace aérien depuis la Seconde Guerre mondiale, avec des conséquences financières importantes, les VAAC ont amélioré leur fonctionnement et, parallèlement, des seuils de concentration de cendres à ne pas dépasser ont été établis. Les seuils d'alerte et seuils de risque ont été fixés à $200 \mu \mathrm{g} / \mathrm{m}^{3}$ et $2 \mathrm{mg} / \mathrm{m}^{3}$ d'air.

Les satellites sont une composante essentielle de cette surveillance, car la plupart des volcans sont situés dans des zones peu ou pas habitées, ou dans des régions du monde où l'information circule mal. L'article qui suit illustre l'apport du sondeur IASI (Infrared Atmospheric Sounding Interferometer) pour surveiller les panaches de dioxyde de soufre $\left(\mathrm{SO}_{2}\right)$ et de cendres ${ }^{(1)}$.

\section{La mission satellite IASI}

La surveillance de l'espace aérien par satellite est possible depuis une vingtaine d'années grâce aux sondeurs UVvisibles (Carn et al., 2008 ; Rix et al., 2009) qui défilent en orbite polaire. Plus récemment, les instruments qui utilisent la bande spectrale de l'infrarouge thermique (Eckhardt et al., 2008 ; Clerbaux et al., 2008) ont permis de compléter la panoplie des mesures disponibles, notamment en fournissant des informations pour les passages de nuit.

Le lancement de l'instrument infrarouge IASI sur le satellite européen MetOp, fin 2006, a considérablement

(1) Pour une présentation plus exhaustive des moyens existants, le lecteur pourra, par exemple, se référer au rapport publié récemment par EUMETSAT/ESA (Zehner et al., 2010) et disponible à l'adresse : http://earth.eo.esa.int/workshops/ Volcano/files/STM_280_ash101124.pdf.

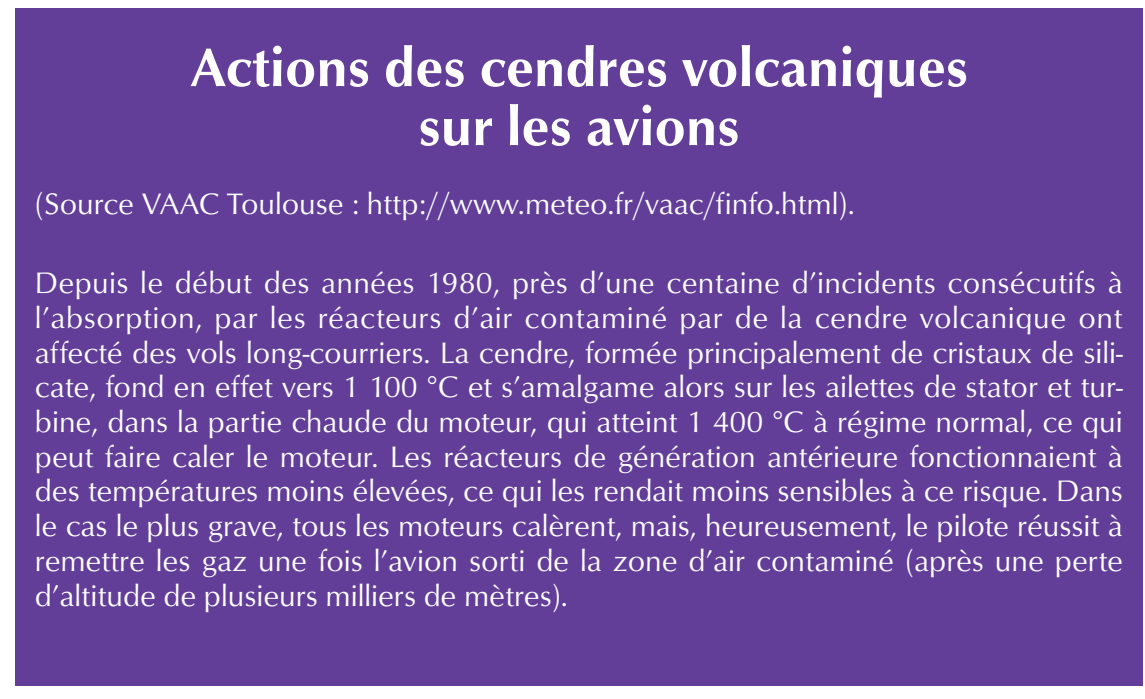

amélioré la possibilité d'obtenir des informations en temps quasi réel pour le suivi de la composition de l'atmosphère aux échelles globales et régionales. La mission IASI est destinée à fournir des mesures atmosphériques pour améliorer les prévisions météorologiques et surveiller les concentrations des gaz qui ont un impact environnemental et climatique (Phulpin et al., 2011 ; Clerbaux et al., 2009). Le sondeur utilise la région spectrale de l'infrarouge thermique (entre 645 et $2760 \mathrm{~cm}^{-1}$ ) pour mesurer les concentrations d'une vingtaine de composés (Coheur et al., 2009 ; Clarisse et al., 2011a), dont le dioxyde de soufre $\left(\mathrm{SO}_{2}\right)$ et les cendres émises lors d'éruptions volcaniques (Clarisse et al., 2008, 2010b). Pour certaines éruptions les mesures d'acide sulfurique $\left(\mathrm{H}_{2} \mathrm{SO}_{4}\right)$ et de sulfure d'hydrogène $\left(\mathrm{H}_{2} \mathrm{~S}\right)$ sont également possibles (Karagulian et al., 2010 ; Clarisse et al., 2011b).

L'instrument est un sondeur passif composé d'un spectromètre à transformée de Fourier lié à un système de détection d'images. La plateforme météorologique $M e t O p$ est une mission d'EUMETSAT, l'organisation européenne pour l'exploitation des satellites météorologiques, qui a pris en charge le lancement et contrôle les opérations en orbite, tandis que le Centre national d'études spatiales (CNES) s'est occupé de la conception et de la maîtrise d'œuvre de l'instrument. Le premier sondeur IASI a été lancé fin octobre 2006, et fournit quotidiennement $15 \mathrm{~Gb}$ de données (plus de 1,2 millions d'observations) qui sont exploitées par les centres météorologiques et par les scientifiques. Le lancement du prochain IASI est prévu en avril 2012, suivi d'un troisième en 2016.
Le satellite $\mathrm{Met} O p$ vole sur une orbite polaire et traverse le plan équatorial à 9 h 30, heure locale. La durée d'une orbite est d'environ 101 minutes, ce qui implique que le satellite fait approximativement 14 révolutions par jour. IASI observe la surface terrestre avec une matrice de $2 \times 2$ pixels de $12 \mathrm{~km}$ de diamètre chacun, et a une excellente couverture horizontale liée à sa capacité à sonder l'atmosphère en mode balayage, perpendiculairement à la trace, avec une amplitude totale de 2050 kilomètres à la surface de la Terre (figure 2).

Le signal fourni par l'instrument est un spectre qui contient les signatures en émission et en absorption des molécules qui se trouvent le long du trajet optique entre la surface de la Terre et le détecteur. Les spectres sont obtenus avec une qualité radiométrique élevée (inférieure à $0,1 \mathrm{~K}$ dans la zone

Figure 2 - Sondage effectué par l'instrument IASI à bord du satellite MetOp : I'instrument balaie la surface de la Terre perpendiculairement à sa trace, avec un angle de $48^{\circ}$ de part et d'autre du nadir (30 vues au total). Chaque scène est observée par 4 empreintes au sol de $12 \mathrm{~km}$ de diamètre simultanément. Le signal analysé est un spectre atmosphérique qui contient les absorptions caractéristiques des molécules qui sont présentes le long du trajet optique.

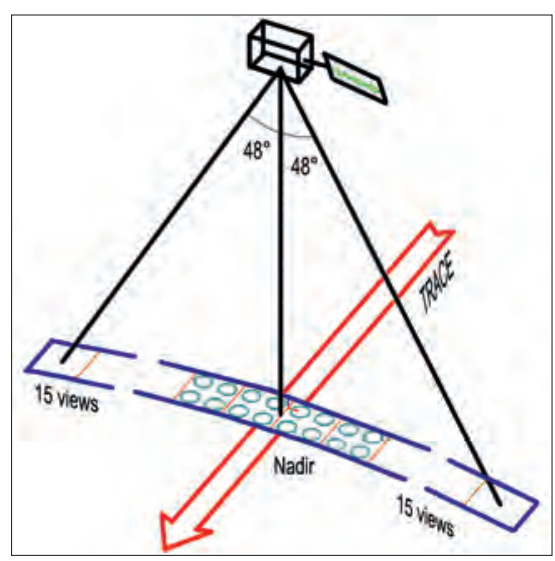


d'absorption du $\mathrm{SO}_{2}$, pour une scène à $280 \mathrm{~K}$ ) et avec une résolution spectrale de $0,5 \mathrm{~cm}^{-1}$ constante sur la région spectrale couverte par l'instrument. Ils sont exprimés en luminance spectrale $\left(\mathrm{W} /\left(\mathrm{cm}^{2} \cdot \mathrm{sr}_{\mathrm{cm}} \mathrm{cm}^{-1}\right)\right)$. Pour certaines études, il peut être nécessaire de les convertir en température de brillance (kelvin), pour mettre en évidence les variations de la ligne de base du spectre. L'analyse de ces spectres atmosphériques, en tenant compte des propriétés spectroscopiques propres à chaque molécule, permet de calculer des concentrations sous forme de colonnes intégrées ou de profils de concentration. L'instrument permet une surveillance continue de la composition atmosphérique avec une couverture globale deux fois par jour. IASI a une sensibilité élevée pour sonder les altitudes entre 5 et $15 \mathrm{~km}$, qui sont aussi les couches atmosphériques affectées par les émissions de gaz et de particules consécutives aux éruptions, et les altitudes de croisière des avions de ligne.

\section{Le système d'alerte automatique}

Chaque volcan est unique et les quantités de dioxyde de soufre et de cendres émises varient ainsi que leur hauteur d'injection dans l'atmosphère. Le $\mathrm{SO}_{2}$ est souvent émis avant l'éruption et peut donc servir d'indicateur pour avertir d'une éruption imminente. Les éruptions peuvent être accompagnées de cendres. Les plus grosses particules (diamètre supérieur à 10-20 $\mu \mathrm{m}$ ) sédimentent rapidement. Les autres peuvent ensuite être transportées. Elles ne sont pas forcément injectées au même niveau que le $\mathrm{SO}_{2}$ et, en cas de fort cisaillement de vent, les directions de transport peuvent être différentes. Une fois dans l'atmosphère, le panache de $\mathrm{SO}_{2}$ va être oxydé en $\mathrm{H}_{2} \mathrm{SO}_{4}$, tandis que le panache de cendres va finir par sédimenter, entraîné par les pluies.

Un système d'alerte automatique a ainsi été mis au point pour détecter dans les spectres IASI :

1) la présence de raies de $\mathrm{SO}_{2}$;

2) la présence de structures caractéristiques des absorptions de cendres volcaniques.

Pour chaque balayage de l'instrument, un ensemble limité de canaux spectraux est analysé pour déclencher l'alerte si un seuil est dépassé. Ce dernier est établi sur la base de différences de

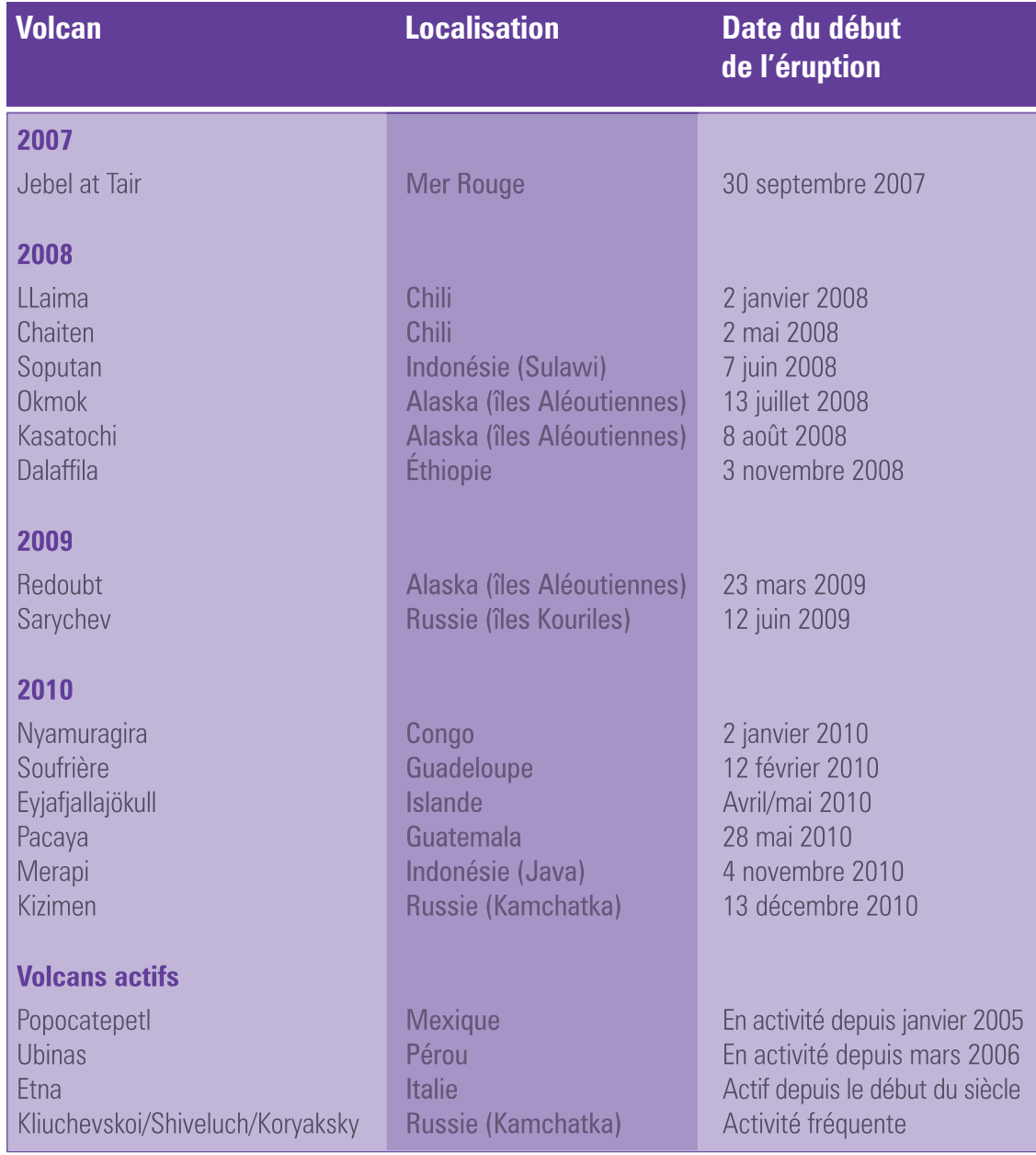

Tableau 1 - Principales éruptions volcaniques détectées par le sondeur infrarouge IASI durant la période 2007-2010.

températures de brillance observées pour les canaux sélectionnés. Le système fonctionne automatiquement et en continu, et envoie une alerte par courriel chaque fois qu'au moins un pixel dépasse le seuil d'alerte (fixé à $2 \mathrm{~K}$ pour $\mathrm{SO}_{2}$ ). L'alerte renseigne le nombre de pixels dépassant le seuil, leur localisation géographique, et l'amplitude de la différence de température mesurée pour chaque pixel. Ces alertes $\mathrm{SO}_{2}$ sont répertoriées et disponibles pour tous (avec une projection sur une carte qui permet de localiser l'événement) sur le site Web de l'Université libre de Bruxelles (ULB) : http://cpm-ws4. ulb.ac.be/Alerts/index.php.

Depuis la mise en œuvre du système, des dizaines d'éruptions volcaniques ont été détectées (voir tableau 1 pour une liste complète), et aucune fausse alerte n'a été signalée. Ces alertes constituent l'un des éléments mis à la disposition de la communauté pour décider du déplacement éventuel des populations. Le $\mathrm{SO}_{2}$ étant souvent émis avant le déclenchement d'écoulements de lave, il permet parfois de donner des alertes d'éruption imminente, comme par exemple dans le cas de l'éruption du
Mérapi (Indonésie). Ce volcan est entré en éruption le 26 octobre 2010. Il a alors expulsé des matériaux, des cendres et de la lave à plusieurs reprises sous forme de coulées pyroclastiques et d'un nuage de cendres qui s'est élevé à $1,5 \mathrm{~km}$ d'altitude. La veille, le gouvernement indonésien, sur base des informations fournies par l'instrument IASI, augmentait le niveau d'alerte au rang maximum, entamant une évacuation de plus de 40000 personnes dans un rayon de $10 \mathrm{~km}$ et sauvant des milliers de vies (Fred Prata, communication privée). Les paragraphes ci-dessous fournissent plus de détails sur les canaux spectraux utilisés et sur la méthode mise en œuvre pour suivre les déplacements des panaches de cendres et de $\mathrm{SO}_{2}$.

\section{Mesure du $\mathrm{SO}_{2}$}

La méthode de détection du $\mathrm{SO}_{2}$ et la mesure de concentration ont été mises au point en effectuant des études de sensibilité sur les paramètres spectroscopiques associés au $\mathrm{SO}_{2}$, les concentrations et les altitudes d'injection du $\mathrm{SO}_{2}$ dans l'atmosphère lors d'une éruption (Clarisse et al., 2008). La présence 


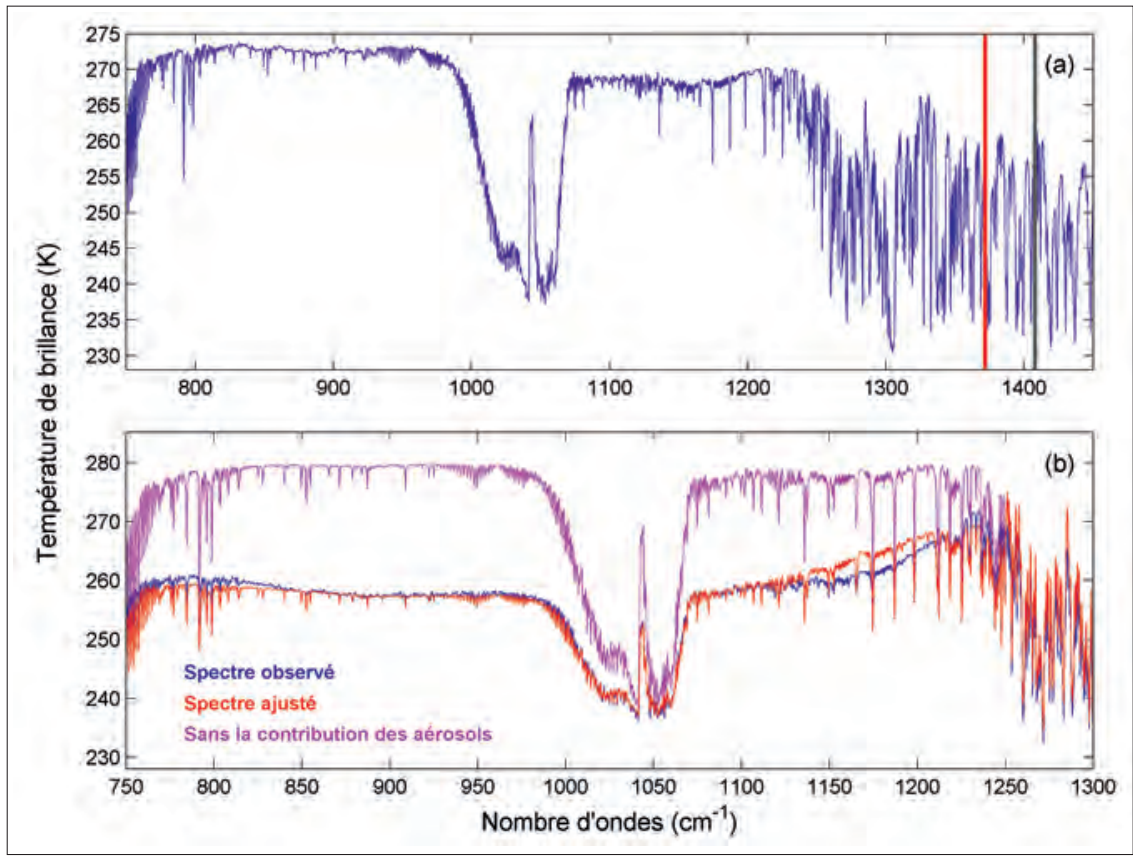

de $\mathrm{SO}_{2}$ est établie en utilisant un filtre basé sur des différences de températures de brillance calculées pour 4 canaux spectraux IASI. Les deux premiers canaux sont choisis pour caractériser la ligne de base et correspondent à des canaux « propres » (sans $\mathrm{SO}_{2}$ et avec un minimum d'absorption d'autres gaz) : 1407,25 et $1408,75 \mathrm{~cm}^{-1}$. Les deux autres canaux sont choisis dans la bande d'absorption la plus sensible au $\mathrm{SO}_{2}$ (1 371,50 et $1371,75 \mathrm{~cm}^{-1}$ ) et minimisent les interférences avec l'eau, qui absorbe dans la même région spectrale. Quand la différence entre les canaux propres et les canaux sensibles au $\mathrm{SO}_{2}$ atteint $2 \mathrm{~K}$, l'alerte automatique se déclenche. La figure 3 représente un spectre atmosphérique typique d'une éruption, sur lequel apparaissent les raies d'absorption du $\mathrm{SO}_{2}$ (mélangées à celles de la vapeur d'eau). Les canaux utilisés pour la détection sont indiqués. La figure 4 montre les régions du globe pour lesquelles l'alerte $\mathrm{SO}_{2}$ a été donnée pour trois jours de juin 2011.

Les différences de températures de brillance ont été estimées au préalable pour différentes combinaisons d'altitudes du panache et de concentrations de $\mathrm{SO}_{2}$. Les différences de températures observées qui ont permis la détection $\mathrm{du} \mathrm{SO}_{2}$ peuvent ensuite être converties en concentrations (pour le $\mathrm{SO}_{2}$ l'unité utilisée est l'unité Dobson, DU(1) en faisant une hypothèse sur l'altitude du panache. L'altitude du panache peut être estimée à partir de simulations, en

(1) Un DU correspond à une couche qui aurait une épaisseur de 10 micromètres dans les conditions standard de température et de pression. faisant le rapport entre le spectre étudié et un spectre de référence, ou à partir d'informations fournies par d'autres sondeurs (par exemple, le lidar CALIOP à bord du Winker et al., 2009), ou par des modèles de trajectoires.

\section{Mesure des cendres}

Les cendres volcaniques absorbent l'infrarouge dans la région spectrale de la fenêtre atmosphérique (entre 800 et $1200 \mathrm{~cm}^{-1}$ environ), voir figure 3. La ligne de base est affectée par ces absorptions, et la signature spectrale observée est fonction de la taille des particules, de leur composition, et de l'altitude d'émission du panache (Pierangelo et al., 2004 ; Clarisse et al., 2010a). Pour la détection et une estimation de la concentration des cendres, la méthode se fonde sur une comparaison entre satellite CALIPSO, $\angle$ Figure 3 - Spectres IASI (en bleu) mesurés dans une zone riche en $\mathrm{SO}_{2}$ (a) et dans une région riche en cendres (b). Les raies d'absorptions caractéristiques des molécules présentes sur le trajet optique $\mathrm{H}_{2} \mathrm{O}, \mathrm{CO}_{2}$ et $\mathrm{O}_{3}$ essentiellement) sont présentes. La « ligne de base » correspond à la température de surface de la scène, autour de $273 \mathrm{~K}$ pour la figure (a). Les canaux spectraux utilisés pour la détection systématique du $\mathrm{SO}_{2}$ (voir texte et (a)) sont indiqués par des lignes verticales. Des spectres simulés sont aussi représentés, pour montrer que la présence de particules affecte la ligne de base et se présente sous la forme d'un continuum. Ces spectres ont été calculés en utilisant un code de transfert radiatif avec (en rouge) et sans (en rose) aérosols.

les spectres mesurés et un jeu de spectres de référence, sélectionnés lors d'éruptions volcaniques passées (Clarisse et al., 2010b). Une relation linéaire est recherchée entre le spectre mesuré et les spectres de référence. Si les coefficients de cette relation linéaire sont dans un intervalle acceptable, défini au préalable par des études de sensibilité, le

VFigure 4 - Différences de températures de brillance mesurées par IASI qui caractérisent la présence de $\mathrm{SO}_{2}$ pour les 6,14 et 17 juin 2011 lors du passage du satellite le matin. Les traces des éruptions des volcans Grímsvötn (Islande), Cordón Caulle (Chili) et Nabro (Érythrée) sont visibles.

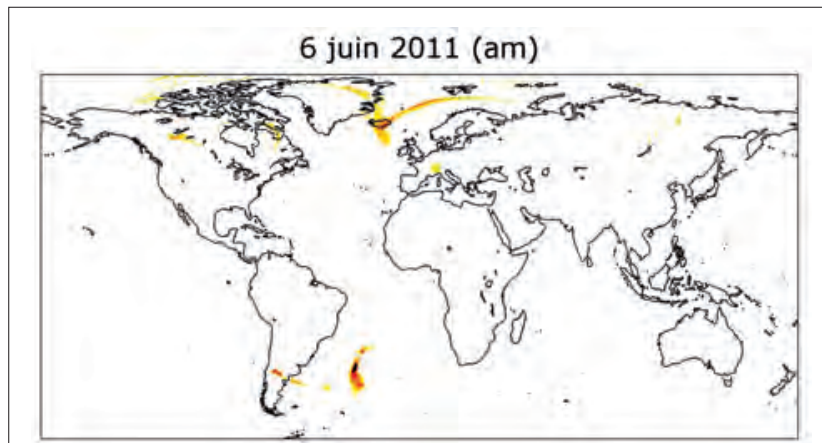

14 juin 2011 (am)

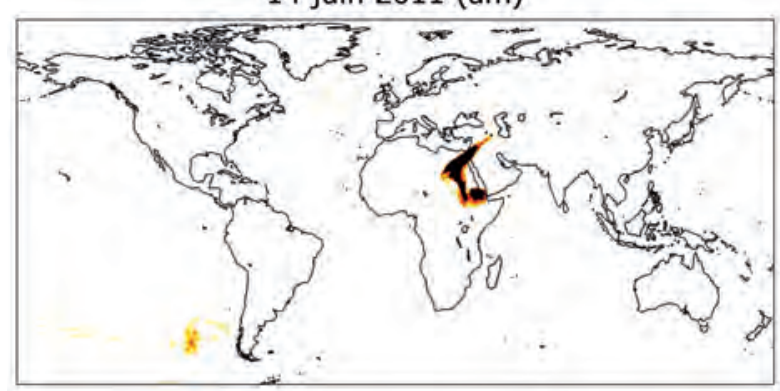

17 juin 2011 (am)

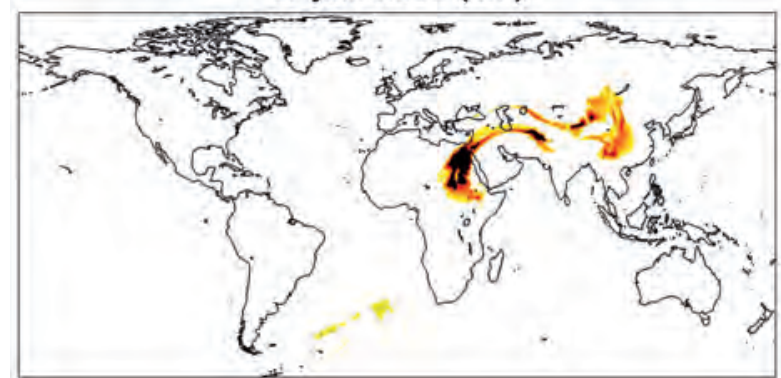

$\begin{array}{llllllllll}0.5 & 1 & 1.5 & 2 & 2.5 & 3 & 3.5 & 4 & 4.5 & 5\end{array}$

$\mathrm{SO}_{2}$ (DBT) 
spectre est identifié comme contaminé par des cendres. Les coefficients sont calculés à partir des spectres en températures de brillance et en utilisant un ensemble de 77 canaux entre 770 et $1240 \mathrm{~cm}^{-1}$, sélectionnés pour éviter les interférences avec des gaz traces absorbant dans cette région spectrale.

\section{Suivi des panaches volcaniques, mai-juin 2011}

Chaque année plusieurs éruptions sont détectées par IASI. Leur impact sur l'aviation varie selon la zone géographique affectée, l'altitude d'injection du $\mathrm{SO}_{2}$, la présence ou non de cendres, et la persistance de l'événement. Les mois de mai et juin 2011 ont été marqués par une succession de trois éruptions volcaniques, mettant en alerte continue les centres responsables de la surveillance des espaces aériens. L'instrument IASI a permis de suivre en détails ces trois éruptions (voir figure 4). Les estimations actuelles indiquent qu'elles ont injecté une masse totale de 2-3 $\mathrm{Tg} \mathrm{SO}_{2}$ dans l'atmosphère.

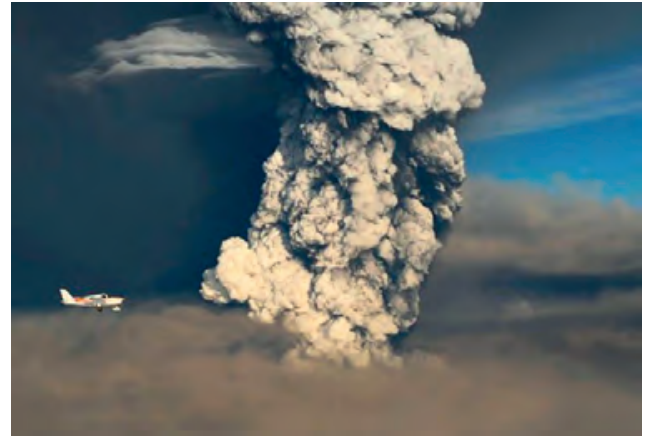

composante qui descend vers le sud et atteindra le nord de l'Allemagne, et une composante qui suit celle de $\mathrm{SO}_{2}$. Le retentissement médiatique de cette éruption a été important, suite à la perturbation majeure engendrée un an auparavant par l'éruption du volcan Eyjafjallajökull. L'éruption du Grímsvötn a été plus intense que celle de Eyjafjallajökull mais s'est réduite

Photo 1 - Passage d'un avion à côté du nuage de cendres de l'éruption du volcan islandais Grimsvötn le 21 mai 2011 (C) Olafur Sirgurjónsson)

\section{Grímsvötn (Islande)}

Grímsvötn est le volcan le plus actif d'Islande (voir photo 1). L'éruption a débuté le 20 mai 2011 ; le panache de cendres a perturbé les opérations de vol en Islande et dans le nord de l'Allemagne. La figure 5 illustre le suivi par IASI des panaches de $\mathrm{SO}_{2}$ et de cendres transportés par les vents les 22 et 23 mai. Dans ce cas, on peut voir que les panaches de $\mathrm{SO}_{2}$ et de cendres ont été portés par les vents dans des directions opposées : le $\mathrm{SO}_{2}$ est transporté vers le nord et s'étend rapidement du Groenland jusqu'en Scandinavie, tandis que le panache de cendre se divise en une rapidement. En effet, la nature différente du magma associée à des vents dominants, qui ne portaient pas le panache vers l'Europe, ont limité l'impact de l'événement sur le trafic aérien. Le magma de Eyjafjallajökull était de type explosif et a engendré la création de particules de cendres très fines (90\% des particules $<1 \mathrm{~mm}$ ), dispersées ensuite à travers l'Europe par les vents de nord-ouest dominants liés à l'anticyclone des Açores. Grímsvötn a engendré des particules de taille plus importante, qui sont retombées plus rapidement au sol.

$\nabla$ Figure 5 - Panaches de $\mathrm{SO}_{2}$ (en haut) et de cendres (en bas) mesurés par IASI le lendemain et le surlendemain de l'éruption du volcan Grimsvötn (triangle bleu) Les panaches ont été transportés dans des directions opposées. Les spectres représentés sur la figure 3 ont été sélectionnés au cœur des panaches.

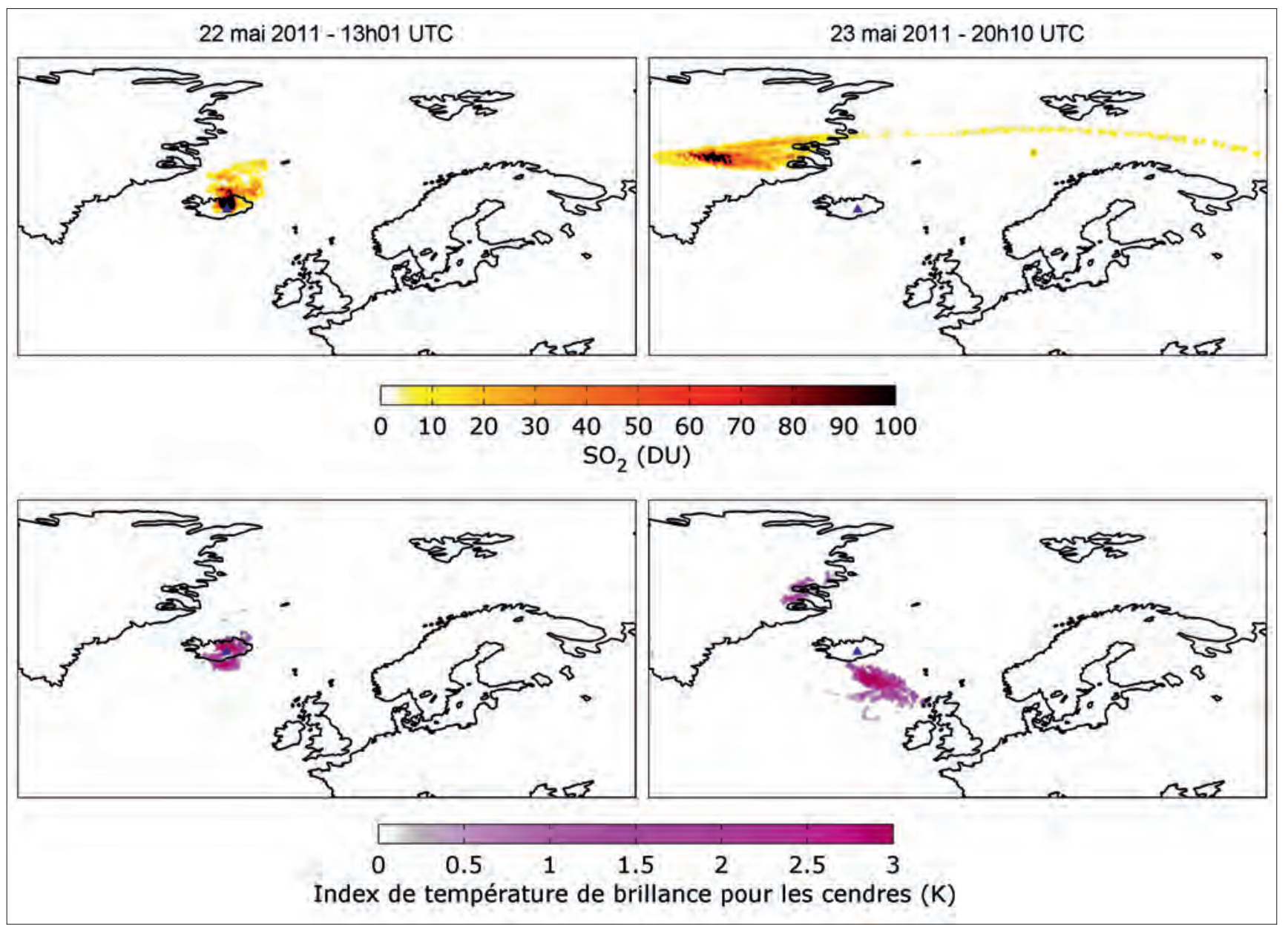




\section{Cordón Caulle (Chili)}

Le 4 juin, avant que les traces du volcan islandais n'aient disparu (voir figure 4), l'éruption du volcan Puyehue-Cordón Caulle a envoyé des nuages de cendre dans la haute atmosphère à une altitude d'environ 15 kilomètres. Des zones étendues autour du volcan et jusqu'en Argentine ont été couvertes de cendres (voir photo 2). La figure 6 illustre le déplacement du $\mathrm{SO}_{2}$ au cours des jours qui ont suivi la première éruption (dans ce cas il semblerait que les panaches cendres et $\mathrm{SO}_{2}$ aient circulé ensemble) : son trajet démarre du Chili le 4 juin, il atteint l'Australie le 11, retourne au Chili le 15 et repasse au-dessus de l'Australie le 20 juin. Le panache de cendres, composé de particules fines, a été transporté par un vent très rapide (jet stratosphérique) et a fait quatre fois le tour de la Terre ${ }^{(1)}$. Le trafic aérien a été perturbé dans toute la région et plusieurs VAAC ont été mobilisés pour son suivi.

\section{Nabro (Érythrée)}

Pendant que les équipes continuaient à suivre le voyage du panache chilien, une autre éruption s'est produite en Érythrée, durant la nuit du 12 juin 2011 (voir figure 4). C'est la première fois qu'une éruption est rapportée pour ce volcan. Des quantités très importantes de cendres et de gaz ont été émises, et certains satellites indiquent une quantité record (depuis qu'on dispose de mesures satellites) de $\mathrm{SO}_{2}$. Les panaches ont ensuite dérivé sur une grande partie de

Photo 2 - La station de montagne Villa la Angostura (en Argentine) recouverte de cendres provenant du volcan chilien Puyehue-Cordón Caulle. (C) Osvaldo Peralta)

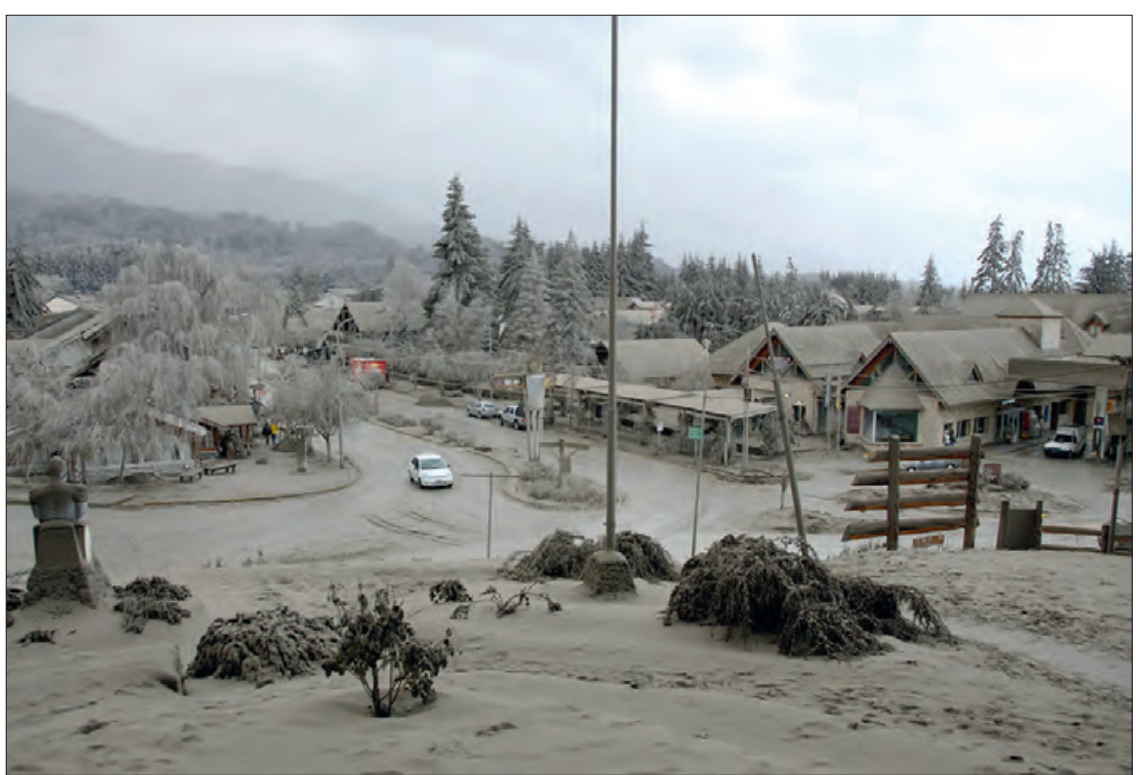

volcan est situé en zone habitée, la détection d'émissions de gaz avant l'explosion du volcan permet parfois de donner l'alerte aux populations locales. Certains volcans se trouvent dans des régions qui sont traversées quotidiennement par des avions de ligne. Il est maintenant reconnu que les panaches de cendres abîment les moteurs et réduisent la visibilité, et que l'acide sulfurique (qui provient du $\mathrm{SO}_{2}$ oxydé dans l'atmosphère) endommage les équipements. L'épisode du volcan islandais Eyjafjallajökull et les conséquences sociétales engendrées par cet événement ont permis aux scientifiques de s'organiser pour proposer d'établir un réseau de surveillance qui s'appuie à la fois sur des instruments basés au sol et à bord de satellite.

Les satellites ont apporté une contribution déterminante pour la surveillance des éruptions volcaniques et pour le suivi de la contamination par les cendres de l'espace aérien. Si le

Le sondeur IASI, qui vole à bord du satellite MetOp depuis fin 2006, a apporté une contribution importante au système de surveillance mis en place à l'échelle du globe. Un système d'alerte automatique de détection de $\mathrm{SO}_{2}$ fonctionne continuellement pour surveiller la présence ou l'éminence d'une éruption. Par rapport à d'autres sondeurs disponibles, cet instrument apporte des informations en quasi temps réel (moins de 3 heures après le passage du satellite), avec une excellente couverture géographique et la possibilité de mesurer de nuit (car il est basé sur de l'instrumentation qui fonctionne dans l'infrarouge), et avec une résolution spatiale assez bonne $(12 \mathrm{~km})$. En revanche, il a une sensibilité limitée (il faut que les concentrations de $\mathrm{SO}_{2}$ dépassent $1 \mathrm{DU}$ pour être détectées)

(1) Une animation produite à partir d'images des satellites géostationnaires Meteosat est disponible à l'adresse : http://www.eumetsat.int/Homel Main/News/Features/808272?l=en. 
et l'estimation de l'altitude du panache est imprécise. Ces informations sont fournies par d'autres sondeurs et alimentent les modèles atmosphériques (Haywood et al., 2010) qui modélisent et prédisent les déplacements des panaches de cendres.

Les travaux de ces dernières années ont également permis de mettre en œuvre la détection de cendres à partir des spectres IASI, mais la mise en place d'un système d'alerte automatique est compliquée par le fait que les particules de cendres sont différentes pour chaque éruption, et que d'autres événements comme les feux et les tempêtes de sable sont aussi susceptibles de transporter des particules sur des milliers de kilomètres. Nous travaillons actuellement sur des tests multicritères qui permettront de différencier ces événements.

Ces mesures viennent compléter un dispositif plus complet qui comprend des lidars (sol ou embarqués) - qui permettent d'avoir des informations sur la verticale -, des mesures locales, ainsi que d'autres mesures satellites basées sur d'autres techniques. Dans le futur, avec un instrument IASI amélioré, comme par exemple le concept IASING actuellement en cours de définition au CNES, les mesures seraient encore plus précises, le seuil de détection plus élevé, et d'autres gaz éjectés par les éruptions (par exemple, $\mathrm{H}_{2} \mathrm{~S}$ ) pourraient être plus facilement détectés et mesurés.

\section{Remerciements}

Les chercheurs du LATMOS remercient le CNES qui soutient financièrement les activités scientifiques autour de la mission IASI depuis de nombreuses années, en particulier Carole Deniel, Carole Larigauderie et Thierry Phulpin pour les discussions scientifiques fréquentes et enrichissantes. Une partie de nos travaux s'appuie sur la base de données atmosphériques Ether (http://ether.ipsl. jussieu.fr) et nous remercions Cathy Boonne et Nathalie Poulet-Crovisier pour leur aide. La recherche en Belgique est menée grâce aux fonds FRS-FNRS et aux contrats ESA-Prodex du Belgian State Federal Office for Scientific, Technical and Cultural Affairs.

\section{Bibliographie}

\footnotetext{
- Carn S. A., A. J. Krueger, N. A. Krotkov, K. Yang et K. Evans, 2008 : Tracking volcanic sulfur dioxide clouds for aviation hazard mitigation. Natural Hazards, DOl:10.1007/s11069-008-9228-4.

- Clarisse L., P.-F. Coheur, A. J. Prata, D. Hurtmans, A. Razavi, T. Phulpin, J. Hadji-Lazaro et C. Clerbaux, 2008 : Tracking and quantifying volcanic SO2 with IASI - the September 2007 eruption at Jebel at Tair. Atmos. Chem. Phys., 8, 7723-7734.

- Clarisse L., D. Hurtmans, A. J. Prata, F. Karagulian, C. Clerbaux, M. De Mazière et P. F. Coheur, 2010a : Retrieving radius, concentration, optical depth, and mass - of different types of aerosols from high resolution infrared nadir spectra. Appl. Opt., 49, 3713-3722.

- Clarisse L., F. Prata, J.-L Lacour, D. Hurtmans, C. Clerbaux et P.-F. Coheur, 2010b : A correlation method for volcanic ash detection using hyperspectral infrared mea- surements. Geophys. Res. Lett., 37, L19806, D0I:10.1029/2010GL044828.

- Clarisse L., Y. R'Honi, P.-F. Coheur, D. Hurtmans et C. Clerbaux, 2011 a : Thermal infrared nadir observations of 24 atmospheric gases. Geophys. Res. Lett., 38 , : L10802, D0I:10.1029/2011GL047271.

- Clarisse L., P.-F. Coheur, S. Chefdeville, J.-L. Lacour, D. Hurtmans et C. Clerbaux, $2011 \mathrm{~b}$ : Infrared satellite observations of hydrogen sulfide in the volcanic plume of - the August 2008 Kasatochi eruption. Geophys. Res. Lett., 38, L10804, D0I:10.1029/2011GL047402.

- Clerbaux C., P.-F. Coheur, L. Clarisse, J. Hadji-Lazaro, D. Hurtmans, S. Turquety, K. Bowman, H. Worden et S. A. Carn, 2008 : Measurements of S02 profiles in - volcanic plumes from the NASA Tropospheric Emission Spectrometer (TES). Geophys. Res. Lett., 35, L22807, D0I:10.1029/2008GL035566.

- Clerbaux C., A. Boynard, L. Clarisse, M. George, J. Hadji-Lazaro, H. Herbin, D. Hurtmans, M. Pommier, A. Razavi, S. Turquety, C. Wespes et P.-F. Coheur, - 2009 : Monitoring of atmospheric composition using the thermal infrared IASI/MetOp sounder. Atmos. Chem. Phys. , 9, 6041-6054.

- Coheur P.-F., L. Clarisse, S. Turquety, D. Hurtmans et C. Clerbaux, 2009 : IASI measurements of reactive trace species in biomass burning plumes. Atmos. Chem. Phys., 9, 5655-5667.

- Eckhardt S., A. J. Prata, P. Seibert, K. Stebel et A. Stohl, 2008 : Estimation of the vertical profile of sulfur dioxide injection into the atmosphere by a volcanic eruption - using satellite column measurements and inverse transport modelling. Atmos. Chem. Phys., 8, 3881-3897, D0I:10.5194/acp-8-3881-2008.
}

- Haywood J. M., A. Jones, L. Clarisse, A. Bourassa, J. Barnes, P. Telford, N. Bellouin, O. Boucher, P. Agnew, C. Clerbaux, P. Coheur, D. Degenstein et

P. Braesicke, 2010 : Observations of the eruption of the Sarychev volcano and simulations using the HadGEM2 climate model. J. Geophys. Res., 115, D21212 DOl:10.1029/2010JD014447.

- Karagulian F., L. Clarisse, C. Clerbaux, A. J. Prata, D. Hurtmans et P.-F. Coheur, 2010 : Detection of volcanic SO2, ash, and H2SO4 using the Infrared Atmospheric - Sounding Interferometer (IASI). J. Geophys. Res., 115, D00L02, DOI:10.1029/2009JD012786.

- Phulpin T., C. Camy-Peyret, J. Taylor, C. Clerbaux, P. Coheur, C. Crevoisier, D. Edwards, A. Gambacorta, V. Guidard, F. Hilton, N. Jacquinet, R. Knuteson, L. Lavanant, T. McNally, M. Matricardi, H. Revercomb, C. Serio, L. Strow, P. Schlüssel, D. Klaes et C. Larigauderie, 2011 : Les résultats exceptionnels de lasi - sondeur atmosphérique hyperspectral de Metop. La Météorologie, $8^{\text {e }}$ série, 72, $19-30$.

- Pierangelo C., A. Chédin et P. Chazette, 2004 : Measurements of stratospheric volcanic aerosol optical depth from NOAA TIROS Observational Vertical Sounder (TOVS) - observations. J. Geophys. Res., 109, D03207, D0I:10.1029/2003JD003870.

- Rix M., P. Valks, N. Hao, D. Loyola, W. Zimmer, S. Emmadi, T. Erbertseder, J. van Geffen, L. Clarisse, C. Clerbaux et P.-F. Coheur, 2009 : Satellite monitoring of - volcanic sulfur dioxide emissions for early warning of volcanic hazards. IEEE Journal of Selected Topics in Applied Earth Observations and Remote Sensing (JSTARS), 2(3), 196-206, DOI:10.1109/JSTARS.2009.2031120.

- Winker D. M., M. A. Vaughan, A. Omar, Y. Hu, K. A. Powell, Z. Liu, W. H. Hunt et S. A. Young, 2009 : Overview of the CALIPSO Mission and CALIOP Data - Processing Algorithms. J. Atmos. Oceanic Technol., 26, 2310-2323.

- Zehner C. et al., 2010 : Monitoring volcanic ash from space. Proceedings of the ESA-EUMETSAT workshop on the 14 April to 23 May 2010 eruption at the Eyjafjoll volcano, South Iceland; ESA ESRIN 26-27 May 2010. Publication ESA STM-280. D0I:10.5270/atmch-10-01. 